\title{
CONTROLE QUÍMICO DE PLANTAS DANINHAS AQUÁTICAS EM CONDIÇÕES CONTROLADAS - CAIXA D'ÁGUA ${ }^{1}$
}

\author{
DAGOBERTO MARTINS ${ }^{2}$, EDIVALDO D. VELINI ${ }^{2}$, ANDERSON L. CAVENAGHI ${ }^{3}$, CRISTINA G. DE MENDONÇA ${ }^{3}$, \\ CRISTIANE G. DE MENDONÇA ${ }^{3}$
}

\section{RESUMO}

Durante o ano de 1998 foram conduzidos quatro experimentos em Botucatu, Estado de São Paulo, Brasil, em condições de caixas d'água, com o objetivo de estudar o efeito de alguns herbicidas sobre Eicchornia crassipes, Pistia stratiotes, Thypha subulata e Salvinia molesta. Os herbicidas e doses utilizadas foram: imazapyr a 125, 250, $500,1.000,1.500$ e 2.000 g e.a./ha; $2,4 \mathrm{D}$ a 1.200 g e.a./ha; glyphosate a 3.360 g e.a./ha. Houve, ainda, uma testemunha sem aplicação de herbicidas. Os experimentos foram instalados no delineamento experimental em blocos ao acaso, com quatro repetições. As parcelas experimentais foram constituídas pelas caixas d'água com dimensões de 60x60x60 cm e colocadas a pleno sol no campo. Utilizou-se 3, 12, 25 e 60 plantas/caixa de $T$. subulata, $P$. stratiotes, E. crassipes e $S$. molesta, respectivamente. Os herbicidas foram aplicados com pulverizador costal a pressão constante de $\mathrm{CO}_{2}$ de $1,75 \mathrm{Bar}$, munido de barra com bicos Teejet 110.02 VS e com um consumo de calda de 200 1/ha. Foram efetuadas avaliações visuais de controle de sete em sete dias por 35 a 49 dias, dependendo da espécie. Todos os herbicidas testados foram eficientes no controle de E. crassipes e o imazapyr foi o único herbicida a promover morte total das plantas, independente da dose utilizada. Os herbicidas 2,4 D e glyphosate não foram eficientes no controle de $P$. stratiotes. O herbicida imazapyr foi eficiente no controle de $P$. stratiotes, exceto na dose de $125 \mathrm{~g}$ e.a./ha. Todos os herbicidas foram eficientes no controle de $T$. subulata, exceção ao imazapyr na dose de125 g e.a./ha. Houve rebrota de T. subulata nas parcelas aplicadas com 2,4 D. Nenhum tratamento químico foi eficiente no controle de S. molesta.

Palavras chave: Planta daninha, imazapyr, 2,4 D, glyphosate.

\section{ABSTRACT}

\section{Chemical aquatic weed control in resevoir}

In 1998 were carried out four trials in Brazil to studied the effect of herbicides on weed aquatic control in reservoir. The herbicides and dosis used were: imzapyr at 125, 250, 500, 1.000, $1.500 \mathrm{e} \quad 2.000 \mathrm{~g} / \mathrm{ha}, \quad 2,4 \mathrm{D}$ at $1.200 \mathrm{~g} / \mathrm{ha}$, glyphosate at $3.360 \mathrm{~g} / \mathrm{ha}$ and a treatment without herbicides applyed. The research was conducted in randomized completely block design with four replications. The plots were constituted by reservoirs with $60 \times 60 \times 60 \mathrm{~cm}$ dimensions. All herbicides and dosis were excellent to $E$. crassipes control. The 2,4 D and glyphosate did not promotion a good control of $P$. stratiotes.

The Imazapyr application showed a excellent control to $P$. stratiotes whem the dosis applyed were above $125 \mathrm{~g} / \mathrm{ha}$, except 2,4 D. All herbicides were efficient to $T$. subulata control, except imazapyr at $125 \mathrm{~g} / \mathrm{ha}$. There was sprouted

\footnotetext{
${ }^{1}$ Recebido para publicação em 17/11/98 e na forma revisada em 9/12/99.

${ }^{2}$ Professor Assistente de Doutor, Departamento de Agricultura e Melhoramento Vegetal. FCA/UNESP, C.P. 237, CEP 18603970, Botucatu, SP/Brasil. E-mail: secdamv@ fca.unesp.br.

${ }^{3}$ Eng. Agrônomo, alunos de Pós-Graduação, Departamento de Agricultura e Melhoramento Vegetal. FCA/UNESP, C.P. 237, CEP 18603-970, Botucatu, SP/Brasil. E-mail: secdamv@fca.unesp.br.
} 
of T. subulata whem 2,4 D was applyed. None of herbicides was efficient to $S$. molesta control.

\section{INTRODUÇÃO}

Uma ampla variedade de plantas aquáticas podem ser encontradas vegetando as margens de rios, lagos e reservatórios ou dentro dos mais diversos ambientes aquáticos, empregando-se diferentes mecanismo de adaptação. Enquanto, algumas espécies apresentam-se enraizadas em rios com fortes correntezas, outras somente podem viver em águas paradas ou estagnadas.

As plantas aquáticas que proliferam nos reservatórios e rios provocam vários inconvenientes, como: acúmulo de lixos e outros sedimentos, proliferação de vetores de doenças, dificuldades de navegação, prejuízos ao turismo regional e a pesca e, nestes casos, podem ser denominadas de daninhas. Estas plantas afetam o transporte de cargas das hidrovias dificultando as rotas de navegação, além de prejuízos às usinas hidrelétricas na geração de energia, aumentandose os custos de manutenção (Marcondes \& Tanaka, 1997).

Dentre as principais plantas daninhas aquáticas podem-se destacar plantas do gênero: Eichhornia, Pistia, Echinochloa, Polygonum, Salvinia, Brachiaria, Typha, Egeria e Cyperus . As plantas daninhas aquáticas flutuantes são as que causam os mais sérios e difundidos problemas a nível mundial. A principal planta daninha aquática no Brasil e no mundo é Eichhornia crassipes (Mart.) Solms (aquapé).

Dentre as plantas flutuantes, destacam-se, ainda, Salvinia molesta D.S. Mitchell (salvínia) e Pistia stratiotes L. (alface d'água) que apresentam várias características, como: capacidade de rápida multiplicação vegetativa; habilidade para regenerar-se a partir de pequenas porções do talo, independência parcial ou completa das estruturas sexuais de reprodução, grande área de tecido fotossintético em proporção ao comprimento da planta, ocupando rapidamente os locais onde incida luz e uma independência das condições do substrato (devido a locomoção da superfície
Key words: Weed, imazapyr, 2,4 D, glyphosate.

d'água). Nos canais de irrigação e de drenagem as plantas aquáticas reduzem a velocidade do fluxo de água, aumentam a infiltração da água no solo e incrementam as perdas por transpiração (Pitelli, 1998).

No Brasil, a tabôa é uma planta aquática muito frequente em margens de lagoas ou represas, reservatórios, canais de irrigação e drenagem. Segundo Kissmann (1991), Thypha subulata Crespo \& Pers-Moreau, é uma planta perene, aquática emergente ou palestre, ocorrendo em várzeas úmidas, nas margens de rios, em canais de irrigação e drenagem e em coleções de água doce pouco profunda. Embora, ainda, sem registro de herbicidas no país, o controle químico da tabôa em outros países, através de herbicidas sistêmicos capazes de atingirem os rizomas, temse mostrado eficiente, tanto do ponto de vista técnico quanto econômico (Kissmann, 1991).

Dentre os possíveis manejos dessas plantas, com a finalidade de reduzir o seu desenvolvimento ou mesmo sua eliminação, temse o controle químico. Os herbicidas de reconhecido efeito sobre aguapé, alface d'água, salvínia e tabôa são pertencentes a diversos grupos como: o das imidazolinonas ( imazapyr) que atua na inibição da enzima acetolactato sintase (ALS) que bloquea a síntese de três aminoácidos: a leucina, a valina e isoleucina; o dos ácidos fenóxiacéticos (2,4-D) que é um regulador de crescimento e, o glyphosate que inibe a síntese de aminoácidos.

O objetivo deste trabalho foi avaliar a eficiência de diferentes herbicidas e doses no controle de plantas de aguapé (E. crassipes), alface d'água ( $P$. stratiotes), salvínia (S. molesta) e de tabôa (T.subulata) sob condições de caixa d'água.

\section{MATERIAIS E MÉTODOS}

Quatro experimentos com plantas daninhas aquáticas foram conduzidos na 
Faculdade de Ciências Agronômicas/UNESP, Campus de Botucatu/SP, no Núcleo de Pesquisas Avançadas em Matologia, onde instalou-se um experimento para cada espécie em estudo.

As plantas daninhas aquáticas estudadas foram: E. crassipes, P. stratiotes, S. molesta e $T$. subulata. As plantas foram cultivadas em caixas de fibra de vidro com as seguintes dimensões: $60 \times 60 \times 60 \mathrm{~cm}$, com 120 litros de água. Foi colocado em cada caixa uma camada de terra de 18 litros. As características químicas da terra utilizada nos diferentes experimentos foram: $\mathrm{pH}$ $\left(\mathrm{CaCl}_{2}\right)=4,5 ; \mathrm{M} . \mathrm{O} .\left(\mathrm{g} / \mathrm{dm}^{3}\right)=27 ; \mathrm{P}, \mathrm{Cu}, \mathrm{Fe}, \mathrm{Mn}, \mathrm{e}$ $\mathrm{Zn}=10,0,26,8,26,48,6$ e $0,9 \mathrm{mg} / \mathrm{dm}^{3}$, respectivamente; $\mathrm{H}+\mathrm{Al}, \mathrm{K}, \mathrm{Ca}, \mathrm{Mg}, \mathrm{SB}$ e $\mathrm{CTC}=$ $52,2,3,20,8,30$ e $82 \mathrm{mmol}_{\mathrm{c}} / \mathrm{dm}^{3}$, respectivamente $\mathrm{e}, \mathrm{V} \%=37$. As características físicas da terra eram: $60 \%$ de argila, $34 \%$ de areia e $6 \%$ de silte. Cada caixa foi adubada com $40 \mathrm{~g}$ da fórmula 4-148 de NPK.

As caixas d'água foram mantidas em condições de pleno sol e utilizou-se 3, 12, 25 e 60 plantas/caixa de $T$. subulata, $P$. stratiotes, E. crassipes e $S$. molesta, respectivamente. Os tratamentos foram dispostos no delineamento experimental em blocos ao acaso, com 4 repetições.

Os tratamentos utilizados nos quatro experimentos com as diferentes espécies foram: imazapyr (Arsenal) a 125, 250, 500,1.000, $1.500 \mathrm{e}$ 2.000 g i.a./ha; 2,4 D (U 46 D Fluid) a 1.200 g e.a./ha; glyphosate (Rodeo) a 3.360 g e.a./ha e uma testemunha sem aplicação de herbicidas.

Os herbicidas foram aplicados em plantas adultas, coletadas em represas. As plantas foram distribuídas de forma uniforme nas caixas d'água. Somente a tabôa foi cultivada nas caixas d'água por um período de 35 dias após o corte de sua parte aérea, sendo à aplicação efetuada na rebrota que em média apresentava $1,0 \mathrm{~m}$ de altura.

A aplicação dos herbicidas foi realizada com um pulverizador costal pressão constante de $\mathrm{CO}_{2}$ a 1,75 Bar e com reservatório para 2 litros de calda. O consumo de calda utilizado foi de 200 1/ha. A barra de aplicação apresentava dois bicos tipo Teejet 110.02 VS, distanciados entre si de $0,50 \mathrm{~m}$.

As condições ambientais durante a aplicação dos herbicidas estão apresentadas na Tabela 1 e, foram divididas em condição inicial, intermediária e final durante o tempo em que se processou a aplicação dos herbicidas que foi de 50 minutos para os experimentos com E. crassipes e $P$. stratiotes e de 35 minutos para o com $S$. molesta. Para o experimento com $T$. subulata dividiu-se o tempo de aplicação, 20 minutos, em condição inicial e final.

TABELA 1. Condições ambientais durante a aplicação dos herbicidas para os diferentes experimentos. Botucatu/SP, 1998.

\begin{tabular}{|c|c|c|c|}
\hline & Temperatura $\left({ }^{\circ} \mathrm{C}\right)$ & Umidade relativa $(\%)$ & Velocidade do vento $(\mathrm{m} / \mathrm{s})$ \\
\hline & \multicolumn{3}{|c|}{ Experimentos com E. crassipes e $P$. stratiotes } \\
\hline Condição inicial & 26 & 74,5 & 0 \\
\hline $\begin{array}{l}\text { Condição } \\
\text { intermediária }\end{array}$ & 29 & 72,0 & 0,3 \\
\hline \multirow[t]{2}{*}{ Condição final } & 32 & 61,0 & 0 \\
\hline & \multicolumn{3}{|c|}{ Experimento com S. molesta } \\
\hline Condição inicial & 18 & 70 & 1,2 \\
\hline $\begin{array}{l}\text { Condição } \\
\text { intermediária }\end{array}$ & 17 & 79 & 1,2 \\
\hline \multirow[t]{2}{*}{ Condição final } & 24 & 84 & 1,2 \\
\hline & \multicolumn{3}{|c|}{ Experimento com $T$. subulata } \\
\hline Condição inicial & 19 & 80 & 1,0 \\
\hline Condição final & 19 & 82 & 1,0 \\
\hline
\end{tabular}


As plantas foram avaliadas visualmente aos 7, 14, 21, 28 e 35 dias após a aplicação (DAA), através de uma escala porcentual de notas, onde zero consistia-se em nenhum controle e 100 $\%$ ao controle total das plantas para as espécies de E. crassipes e $P$. stratiotes; aos 14, 21, 28, 35, 42 e 49 dias para $S$. molesta e, aos 7, 14, 21, 28, 35 e 43 dias para $T$. subulata. As avaliações foram realizadas até o desaparecimento ou estabilização dos sintomas (Sociedade Brasileira da Ciência das Plantas Daninhas, 1995).

Os parâmetros utilizados para $\mathrm{o}$ estabelecimento das notas foram: quantidade de plantas mortas, acúmulo de biomassa, inibição do crescimento, quantidade e uniformidade das injúrias, capacidade de rebrota das plantas.

Os resultados obtidos foram submetidos a análise de variância pelo Teste $\mathbf{F}$ e as médias dos tratamentos foram comparadas pelo teste $\mathbf{t}$ a $5 \%$ de probabilidade.

\section{RESULTADOS E DISCUSSÃO}

Na Tabela 2 estão apresentados os resultados da avaliação de controle sobre as plantas de E. crassipes (aguapé) realizados em diferentes épocas após a aplicação dos herbicidas.

TABELA 2. Porcentagem de controle de E. crassipes (aguapé) aos 7, 14, 21, 28 e 35 dias após aplicação dos herbicidas. Botucatu/SP, 1998.

\begin{tabular}{|c|c|c|c|c|c|c|}
\hline \multirow{2}{*}{ Tratamento } & \multirow{2}{*}{ Dose (g e.a/ha) } & \multicolumn{5}{|c|}{ Dias após aplicação } \\
\hline & & 7 & 14 & 21 & 28 & 35 \\
\hline imazapyr & 125 & $2,75 \mathrm{e}$ & $22,00 \mathrm{e}$ & $90,00 \mathrm{~b}$ & $100 \mathrm{a}$ & $100 \mathrm{a}$ \\
\hline imazapyr & 250 & $11,75 \mathrm{~d}$ & $27,50 \mathrm{e}$ & $86,50 \mathrm{~b}$ & $100 \mathrm{a}$ & $100 \mathrm{a}$ \\
\hline imazapyr & 500 & $15,00 \mathrm{~d}$ & $37,50 \mathrm{~d}$ & $92,50 \mathrm{ab}$ & $100 \mathrm{a}$ & $100 \mathrm{a}$ \\
\hline imazapyr & 1.000 & $29,50 \mathrm{c}$ & $72,00 \mathrm{~b}$ & 98,75 a & $100 \mathrm{a}$ & $100 \mathrm{a}$ \\
\hline imazapyr & 1.500 & $33,75 \mathrm{~b}$ & $69,50 \mathrm{~b}$ & 99,25 a & $100 \mathrm{a}$ & $100 \mathrm{a}$ \\
\hline imazapyr & 2.000 & $32,25 \mathrm{bc}$ & $60,00 \mathrm{c}$ & 98,25 a & $100 \mathrm{a}$ & $100 \mathrm{a}$ \\
\hline $2,4 \mathrm{D}$ & 1.200 & $5,00 \mathrm{e}$ & $99,50 \mathrm{a}$ & 99,50 a & 99,25 a & $97,50 \mathrm{ab}$ \\
\hline glyphosate & 3.360 & $91,50 \mathrm{a}$ & $56,25 \mathrm{c}$ & $58,75 \mathrm{c}$ & $83,75 \mathrm{~b}$ & $96,00 \mathrm{~b}$ \\
\hline testemunha & - & - & - & - & - & - \\
\hline F trat. & & $589,96 * *$ & $96,71 * *$ & $31,08 * *$ & $20,27 * *$ & $2,05^{\mathrm{ns}}$ \\
\hline F bloco & & $0,93^{\mathrm{ns}}$ & $1,61^{\mathrm{ns}}$ & $0,15^{\mathrm{ns}}$ & $0,94^{\mathrm{ns}}$ & $1,08^{\mathrm{ns}}$ \\
\hline C. V. $(\%)$ & & 8,47 & 9,44 & 5,43 & 2,59 & 2,19 \\
\hline d. m.s. & & 3,45 & 7,71 & 7,23 & 3,73 & 3,20 \\
\hline
\end{tabular}

** significativo ao nível de $1 \%$ de probabilidade.

${ }^{\mathrm{ns}}$ não significativo.

Médias seguidas de mesma letra, na coluna, não diferem estatisticamente entre si, pelo teste t $(\mathrm{P}>0,05)$.

Os primeiros sintomas de injúrias iniciaram-se aos 7 dias após a aplicação dos herbicidas, sendo mais evidentes nas caixas tratadas com glyphosate e seguido pelas três maiores doses de imazapyr $(1.000,1.500$ e $2.000 \mathrm{~g}$ a./ha), porém estatisticamente inferiores ao glyphosate. Aos 14 dias após a aplicação, o melhor controle foi obtido com o herbicida 2, 4-D, sendo observado um restabelecimento no crescimento das plantas de aguapé com o aplicação herbicida glyphosate e, todas as doses de imazapyr, evoluiram o seu controle.

Todos os tratamentos testados, com exceção ao glyphosate mostraram controles que variaram de bom a excelente, aos 21 dias após a aplicação dos herbicidas. Aos 28 dias após a aplicação dos herbicidas, todos os tratamentos a base de imazapyr, independente da dose, 
proporcionaram um controle de $100 \%$ das plantas e o herbicida 2,4D evidenciou um controle próximo a $100 \%$.

Aos 35 dias após a aplicação dos herbicidas, os resultados mantiveram-se semelhantes aos observados na avaliação efetuada aos 28 dias, porém com um incremento no controle proporcionado pelo glyphosate.

Joshi (1976) só observou morte completa de plantas de aguapé com a aplicação de 2,4 D em duas épocas, em uma aplicação sequencial, com intervá-lo de 14 dias. Estes resultados corroboram com o trabalho, pois com uma única aplicação a morte das plantas não foi completa.

Outros pesquisadores encontraram resultados semelhantes no controle de plantas de aguapé com a aplicação de glyphosate (GutierrezLopez, 1993) e na aplicação de imazapyr (Mukhopadhyay \& Bhattacharyya, 1985)

$\mathrm{Na}$ Tabela 3 estão apresentados os resultados da avaliação de controle sobre as plantas de $P$. stratiotes (alface d'água) realizados em diferentes épocas após a aplicação dos herbicidas.

TABELA 3. Porcentagem de controle de $P$. stratiotes (alface d'água), aos 7, 14, 21,28 e 35 dias após aplicação dos herbicidas. Botucatu/SP, 1998.

\begin{tabular}{lcccccc}
\hline \multirow{2}{*}{ Tratamento } & \multirow{2}{*}{ Dose (g e.a/ha) } & \multicolumn{5}{c}{ Dias após aplicação } \\
\cline { 3 - 7 } \multicolumn{1}{c}{ imazapyr } & 125 & $30,50 \mathrm{c}$ & $81,75 \mathrm{~b}$ & $91,75 \mathrm{a}$ & $81,25 \mathrm{~b}$ & $52,00 \mathrm{c}$ \\
$\quad$ imazapyr & 250 & $69,00 \mathrm{~b}$ & $93,50 \mathrm{a}$ & $98,25 \mathrm{a}$ & $99,25 \mathrm{a}$ & $97,50 \mathrm{a}$ \\
$\quad$ imazapyr & 500 & $65,75 \mathrm{~b}$ & $94,50 \mathrm{a}$ & $98,25 \mathrm{a}$ & $99,50 \mathrm{a}$ & $99,75 \mathrm{a}$ \\
$\quad$ imazapyr & 1.000 & $62,00 \mathrm{~b}$ & $96,50 \mathrm{a}$ & $99,00 \mathrm{a}$ & $99,75 \mathrm{a}$ & $100 \mathrm{a}$ \\
$\quad$ imazapyr & 1.500 & $73,25 \mathrm{~b}$ & $96,50 \mathrm{a}$ & $100 \mathrm{a}$ & $100 \mathrm{a}$ & $100 \mathrm{a}$ \\
$\quad$ imazapyr & 2.000 & $32,50 \mathrm{c}$ & $89,75 \mathrm{ab}$ & $100 \mathrm{a}$ & $100 \mathrm{a}$ & $100 \mathrm{a}$ \\
$\quad$ 2,4 D & 1.200 & $5,00 \mathrm{~d}$ & $20,50 \mathrm{c}$ & $24,50 \mathrm{~b}$ & $27,00 \mathrm{c}$ & $13,25 \mathrm{~d}$ \\
$\quad$ glyphosate & 3.360 & $91,50 \mathrm{a}$ & $97,00 \mathrm{a}$ & $97,75 \mathrm{a}$ & $93,75 \mathrm{a}$ & $68,00 \mathrm{~b}$ \\
$\quad$ testemunha & - & - & - & - & - & - \\
\hline F trat. & & $23,13 * *$ & $54,35^{* *}$ & $75,34 * *$ & $53,87 * *$ & $75,36 * *$ \\
F bloco & & $1,09^{\mathrm{ns}}$ & $0,62^{\mathrm{ns}}$ & $1,57^{\mathrm{ns}}$ & $1,41 \mathrm{~ns}$ & $4,05 *$ \\
C. V. (\%) & & 21,94 & 8,44 & 6,77 & 7,87 & 9,41 \\
\hline d. m. s. & & 17,33 & 10,39 & 8,83 & 10,14 & 10,92 \\
\hline
\end{tabular}

** significativo ao nível de $1 \%$ de probabilidade.

${ }^{\text {ns }}$ não significativo.

Médias seguidas de mesma letra, na coluna, não diferem estatisticamente entre si, pelo teste t $(\mathrm{P}>0,05)$.

Aos 7 dias após a aplicação dos herbicidas apenas as parcelas tratadas com glyphosate apresentavam controle eficiente das plantas de alface d'água.

Mostraram controle eficiente, aos 14 dias após a aplicação dos herbicidas, as caixas d'água tratadas com imazapyr nas doses de 250, 500, 1.000 e $1.500 \mathrm{~g} / \mathrm{ha}$ e com glyphosate. O tratamento com imazapyr a $125 \mathrm{~g} / \mathrm{ha}$ proporcionou o seu melhor controle aos 21 dias após a aplicação
(91,75 \%), mas houve rebrota das plantas, restabelecendo parte da biomassa nas caixas d'água, atingindo apenas $52 \%$ de controle ao final do experimento, aos 35 dias após a aplicação. A partir dos 21 dias nas doses de 1.500 e $2.000 \mathrm{~g} / \mathrm{ha}$ de imazapyr proporcionaram morte completa das plantas.

A aplicação da maior dose de imazapyr retardou o aparecimento das injúrias iniciais observadas com a aplicação das demais doses. 
Talvez este fato seja devido a uma intoxicação rápida das plantas de alface d'água que provavelmente afetou a absorção e/ou translocação inicial, mas aos 28 dias após a sua aplicação as plantas já estavam mortas.

O herbicida 2, 4-D mostrou-se ineficiente no controle da alface d'água, sendo que houve rebrota das plantas nas caixas aplicadas com este herbicida. O mesmo resultado foi observado por Seth et al. (1973) em um estudo realizado em canais de irrigação da Malásia.

As parcelas tratadas com glyphosate mostrou-se inicialmente eficiente no controle das plantas de alface d'água, porém, já aos 35 dias após aplicação apareceram plantas com rebrotas.

Na Tabela 4 estão apresentados os resultados da avaliação de controle sobre as plantas de salvínia realizados em diversas épocas após a aplicação dos herbicidas.

TABELA 4. Porcentagem de controle de $S$. molesta (salvínia) aos 14, 21, 28, 35, 42 e 49 dias após aplicação dos herbicidas. Botucatu/SP, 1998.

\begin{tabular}{|c|c|c|c|c|c|c|c|}
\hline \multirow{2}{*}{ Tratamento } & \multirow{2}{*}{$\begin{array}{l}\text { Dose (g } \\
\text { e.a/ha). }\end{array}$} & \multicolumn{6}{|c|}{ Dias após aplicação } \\
\hline & & 14 & 21 & 28 & 35 & 42 & 49 \\
\hline imazapyr & 125 & $5,00 \mathrm{~d}$ & $10,00 \mathrm{e}$ & $10,00 \mathrm{e}$ & $9,50 \mathrm{~d}$ & $20,00 \mathrm{a}$ & $20,00 \mathrm{~b}$ \\
\hline imazapyr & 250 & $2,00 \mathrm{~g}$ & $5,00 \mathrm{~g}$ & $6,50 \mathrm{~g}$ & $10,00 \mathrm{~d}$ & $12,00 \mathrm{f}$ & $20,00 \mathrm{~b}$ \\
\hline imazapyr & 500 & $3,00 \mathrm{e}$ & $7,00 \mathrm{f}$ & $8,50 \mathrm{f}$ & $10,00 \mathrm{~d}$ & $15,00 \mathrm{e}$ & $18,75 \mathrm{~b}$ \\
\hline imazapyr & 1.000 & $15,00 \mathrm{c}$ & $15,00 \mathrm{~b}$ & $15,00 \mathrm{c}$ & $15,00 \mathrm{~b}$ & $20,00 \mathrm{c}$ & $23,00 \mathrm{a}$ \\
\hline imazapyr & 1.500 & $17,00 \mathrm{~b}$ & $20,00 \mathrm{a}$ & $20,00 \mathrm{a}$ & $19,50 \mathrm{a}$ & $18,00 \mathrm{~d}$ & $20,00 \mathrm{~b}$ \\
\hline Imazapyr & 2.000 & $3,00 \mathrm{f}$ & $12,00 \mathrm{c}$ & $18,00 \mathrm{~b}$ & $19,00 \mathrm{a}$ & $20,00 \mathrm{~b}$ & $19,50 \mathrm{~b}$ \\
\hline $2,4 \mathrm{D}$ & 1.200 & $0,00 \mathrm{~h}$ & $0,00 \mathrm{~h}$ & $0,00 \mathrm{~h}$ & $0,00 \mathrm{e}$ & $0,00 \mathrm{~h}$ & $0,00 \mathrm{c}$ \\
\hline Glyphosate & 3.360 & $20,00 \mathrm{a}$ & $12,00 \mathrm{~d}$ & $12,00 \mathrm{~d}$ & $12,00 \mathrm{c}$ & $12,00 \mathrm{~g}$ & $20,00 \mathrm{~b}$ \\
\hline Testemunha & - & - & - & - & - & - & \\
\hline F trat. & & $99999,9 * *$ & $99999,9 * *$ & $363,08 * *$ & $142,11 * *$ & $99999,9 * *$ & $223,97 * *$ \\
\hline F bloco & & $0,0^{\mathrm{ns}}$ & $0,00^{\mathrm{ns}}$ & $1,62^{\mathrm{ns}}$ & $0,91^{\mathrm{ns}}$ & $0,00^{\mathrm{ns}}$ & $0,75^{\mathrm{ns}}$ \\
\hline C. V. $(\%)$ & & 0,0 & 0,0 & 6,06 & 8,81 & 0,00 & 5,47 \\
\hline d. m. s. & & 0,00 & 0,00 & 1,00 & 1,53 & 0,00 & 1,42 \\
\hline
\end{tabular}

** significativo ao nível de $1 \%$ de probabilidade.

${ }^{\text {ns }}$ não significativo.

Médias seguidas de mesma letra, na coluna, não diferem estatisticamente entre si, pelo teste $\mathrm{t}(\mathrm{P}>0,05)$.

Todos os hebicidas e doses testadas foram ineficientes no controle de $S$. molesta. O herbicida 2,4 D, além de não ser eficiente no controle, promoveu um estímulo de brotações nas plantas de S. molesta. Após 49 (dias após a aplicação), as avaliações foram encerradas, pois ficaram inconsistentes, houve um desenvolvimento das plantas em todas as parcelas, o que dificultou diferenciar as plantas utilizadas inicialmente no experimento das novas que foram emitidas.

$\mathrm{Na}$ Tabela 5 estão apresentados os resultados da avaliação de controle sobre as plantas de tabôa realizados em diferentes épocas após a aplicação dos herbicidas.

Sintomas iniciais de injúrias nas plantas de tabôa mais severos puderam ser observados já aos 7 dias após a aplicação nas parcelas tratadas com o herbicida imazapyr, nas doses de 1.000, 1.500 e 2.000 g e.a./ha e, com o herbicida 2,4 D. Nos demais tratamentos os sintomas foram bem amenos.

Aos 14 dias após aplicação dos herbicidas, todos os tratamentos químicos proporcionaram incrementos nos sintomas de injúria nas plantas de 
tabôa. Com 21 dias após aplicação dos herbicidas, os sintomas, ainda, apresentaram incrementos e nas parcelas tratadas com imazapyr a 1.000, 1.500 e $\quad 2.000$ g e.a./ha, 2,4 D e glyphosate ocorreram controles que variaram de aceitáveis a bom.

TABELA 5. Porcentagem de controle de $T$. subulata (tabôa) aos $7,14,21,28,35$ e 43 dias após aplicação dos herbicidas. Botucatu/SP, 1998.

\begin{tabular}{cccccccc}
\hline \multirow{2}{*}{ Tratamento } & \multirow{2}{*}{ Dose (g e.a/ha). } & \multicolumn{7}{c}{ Dias após aplicação } \\
\cline { 3 - 8 } & & 7 & 14 & 21 & 28 & 35 & 43 \\
\hline imazapyr & 125 & $4,25 \mathrm{e}$ & $22,75 \mathrm{c}$ & $33,75 \mathrm{c}$ & $38,75 \mathrm{c}$ & $66,25 \mathrm{~b}$ & $69,25 \mathrm{~b}$ \\
imazapyr & 250 & $4,50 \mathrm{e}$ & $27,50 \mathrm{c}$ & $38,75 \mathrm{c}$ & $62,50 \mathrm{bc}$ & $84,50 \mathrm{a}$ & $88,75 \mathrm{a}$ \\
imazapyr & 500 & $21,50 \mathrm{~d}$ & $46,75 \mathrm{bc}$ & $72,00 \mathrm{~b}$ & $86,50 \mathrm{ab}$ & $98,50 \mathrm{a}$ & $99,25 \mathrm{a}$ \\
imazapyr & 1.000 & $40,00 \mathrm{c}$ & $72,5 \mathrm{a}$ & $88,75 \mathrm{ab}$ & $93,25 \mathrm{a}$ & $99,00 \mathrm{a}$ & $100,00 \mathrm{a}$ \\
imazapyr & 1.500 & $48,75 \mathrm{c}$ & $65,00 \mathrm{ab}$ & $89,25 \mathrm{ab}$ & $98,00 \mathrm{a}$ & $100,00 \mathrm{a}$ & $100,00 \mathrm{a}$ \\
imazapyr & 2.000 & $65,00 \mathrm{~b}$ & $67,50 \mathrm{ab}$ & $95,50 \mathrm{a}$ & $99,00 \mathrm{a}$ & $100,00 \mathrm{a}$ & $100,00 \mathrm{a}$ \\
2,4 D & 1.200 & $78,50 \mathrm{a}$ & $80,75 \mathrm{a}$ & $82,50 \mathrm{ab}$ & $87,50 \mathrm{a}$ & $88,75 \mathrm{a}$ & $86,25 \mathrm{a}$ \\
glyphosate & 3.360 & $6,75 \mathrm{e}$ & $74,75 \mathrm{a}$ & $83,25 \mathrm{ab}$ & $83,75 \mathrm{ab}$ & $90,75 \mathrm{a}$ & $91,25 \mathrm{a}$ \\
testemunha & - & - & - & - & - & - & - \\
\hline F trat. & & $86,34 * *$ & $6,80 * *$ & $9,28 * *$ & $5,99 * *$ & $4,27 * *$ & $3,46 *$ \\
F bloco & & $3,588^{\mathrm{ns}}$ & $1,600^{\mathrm{ns}}$ & $1,800^{\mathrm{ns}}$ & $1,87 \mathrm{~ns}$ & $2,67 *$ & $2,53 *$ \\
C. V. $(\%)$ & 18,50 & 29,74 & 21,31 & 20,74 & 12,34 & 12,57 \\
\hline d. m. s. & & 9,16 & 25,01 & 22,86 & 24,75 & 16,50 & 16,98 \\
\hline
\end{tabular}

* significativo ao nível de $5 \%$ de probabilidade.

** significativo ao nível de $1 \%$ de probabilidade.

${ }^{\mathrm{ns}}$ não significativo.

Médias seguidas de mesma letra, na coluna, não diferem estatisticamente entre si, pelo teste t $(\mathrm{P}>0,05)$.

Aos 28 dias após aplicação dos herbicidas, os sintomas continuaram a progredir nas parcelas onde receberam o herbicida imazapyr, com destaque para as doses a partir de $500 \mathrm{~g} / \mathrm{ha}$. Com 35 dias após aplicação dos herbicidas, observou-se que nas parcelas tratadas com o herbicida $2,4 \mathrm{D}$ a porcentagem de controle estabilizou-se ao redor de $88 \%$, reduzindo-se para $86 \%$ aos 43 dias devido a incidência de rebrota nas plantas de tabôa.

Aos 45 dias após a aplicação dos herbicidas, as parcelas tratadas com o herbicida imazapyr a 250, 500, 1.000, 1.500 e $2.000 \mathrm{~g}$ e.a./ha evidenciou controles que variaram de bom a excelente, onde atingiram porcentagens de controle que variaram de $88,7 \%$ a $100 \%$, dependendo da dose testada. Ressalta-se, ainda, que o herbicida glyphosate apresentou umbom controle também da tabôa, mas em porcentagem de controle, inferior ao herbicida imazapyr na maioria de suas doses testadas. Também, Prates et. al. (1996), observaram um bom controle de plantas de tabôa em canais de irrigação com a aplicação do herbicida imazapyr.

\section{LITERATURA CITADA}

GUTIERREZ-LOPES, E. Effects glyphosate on different densities of water hyacinth. J. Aq. P. Manag., v.31, p.255-7, 1993.

JOSHI, N.C. Chemical control of water hyacinth. In: Regional Seminar on Noxious Aquatic Vegetation, 1973. Proceedings, s.l.: s.n., 1976, p.263-4. Abstracts of papers... s.n.t. 
KISSMANN, G.K. Plantas infestantes e nocivas.1ed. São Paulo: Basf S.A, 1991. $608 \mathrm{p}$.

MARCONDES, D.A.S., TANAKA, R.H. Plantas aaquáticas nos reservatórios das usinas hidrelétricas da CESP. CONGRESSO BRASILEIRO DA CIÊNCIA DAS PLANTAS DANINHAS, 21, Caxambu, 1997. Workshorp de Plantas Aquáticas Caxambu: SBCPD, 1997 p.2-4.

MUKHOPADHYAY, S.K., BHATTACHARYYA, M. Evaluation of new herbicide AC 252925 (Arsenal) in controlling water hyacinth and Parthenium weeds. In: Annual Conference of Indian Wociety of Weed Science, 1985. Abstracts of papers... s.n.t.
PITELLI, R.A. Macrófitas aquáticas no Brasil, na condição de problemáticas. Workshop de Controle de Plantas Aquáticas. Brasilia: IBAMA, 1998. p. 12-15.

PRATES, H.T., SILVA, J.B., FERREIRA, G.A.L, LEITE, C.E.P., BATISTA, M.J. Curva de dissipação de imazapyr em água de drenos de irrigação, após aplicação para controle de tabôa. Planta Daninha, n.2, v.14, p.12733, 1996.

SOCIEDADE BRASILEIRA DA CIÊNCIA DAS PLANTAS DANINHAS. Procedimentos para instalação, avaliação e análise de experimentos com herbicidas. Londrina: SBCPD, 1995. 42p. 